Enfoques. Revista de Investigación en Ciencias de la Administración http://doi.org/10.33996/revistaenfoques.v3i9.51 No. 9 | Volumen 3 | Enero - Marzo 2019 http://revistaenfoques.org ISSN: $2016-8219$ pp. $15-31$

\section{GESTIÓN DE MANTENIMIENTO CORRECTIVO EN LAS INSTALACIONES UNIVERSITARIAS PÚBLICAS DE LA COSTA ORIENTAL DEL LAGO}

\author{
CORRECTIVE MAINTENANCE MANAGEMENT IN PUBLIC \\ FACILITIES UNIVERSITY OF EAST COAST LAGO
}

\author{
Alba Loaiza \\ Artículo recibido octubre 2018 | Arbitrado noviembre 2018 | Publicado 01 de enero 2019
}

\section{Resumen}

El presente artículo tiene como finalidad describir la gestión de mantenimiento correctivo en las instalaciones de las universidades públicas que tuvo lugar en la Costa Oriental del Lago. Dicho análisis obedeció a la necesidad que tienen las universidades por adaptar y mejorar los procesos de mantenimiento correspondientes al área de infraestructura. La fundamentación teórica se basó en la descripción de la variable de estudio gestión de mantenimiento correctivo. La investigación fue de tipo descriptiva y su diseño no experimental, de campo y transeccional. Finalmente al analizar la variable de estudio se concluyó que las instalaciones universitarias públicas de la Costa Oriental del Lago necesitan considerar los requerimientos de los trabajadores y de los usuarios, así como aplicar la calidad en sus procesos, e implementar, en sus instalaciones, un plan de mantenimiento preventivo para disminuir así los mantenimientos correctivos.

Palabras clave: Gestión de mantenimiento correctivo; infraestructura; instalaciones; procesos de mantenimiento

\begin{abstract}
The purpose of this article is to describe the management of corrective maintenance in the facilities of the public universities that took place on the East Coast of the Lake. This analysis was due to the need for universities to adapt and improve the maintenance processes corresponding to the infrastructure area. The theoretical basis was based on the description of the corrective maintenance management study variable. The research was descriptive and its design was not experimental, field and transectional. Finally, when analyzing the study variable, it was concluded that public university facilities on the East Coast of the Lake need to consider the requirements of workers and users, as well as apply quality in their processes, and implement, in their facilities, a plan of preventive maintenance to reduce corrective maintenance.
\end{abstract}

Key words: corrective maintenance management; facilities; infrastructure; Maintenance processes

\section{Alba Loaiza}

albaloaiza.104@gmail.com Instituto Universitario de Tecnología de Cabimas, Venezuela.

Ingeniero Mecánico, Magister en Gerencia de Empresa. Profesora de la Universidad Politécnica Territorial del Zulia. Doctorante del doctorado en Ciencias de la Educación de la Universidad Rafael María Baralt 


\section{INTRODUCCIÓN}

Las organizaciones en la actualidad tienen que enfrentar altos niveles de competitividad en todos los sectores, por lo cual se hace imprescindible que sus productos y servicios tengan niveles óptimos de calidad que les permitan asegurar su permanencia en el mercado.

En este sentido, las instituciones de educación universitaria no escapan de esta realidad, donde el aumento de la población estudiantil, especialmente en el sector público, genera la necesidad de incrementar los requerimientos de servicios por mantenimiento de las instalaciones. Es necesario mencionar que esta actividad viene realizándose de forma sistemática, sin sufrir a lo largo del tiempo, modificaciones que se ajusten al nuevo contexto educativo que demanda cada día mayor atención a los estudiantes, en sus diversos horarios y modalidades de estudios. De allí que resulte indispensable aplicar nuevas formas de gestión a los procesos y en este caso específico a los procesos de mantenimiento correctivo de las instalaciones, de forma que logre satisfacer las necesidades de los usuarios y demás partes interesadas.

En efecto, la norma COVENIN 3049-93 (1993), establece la no posibilidad de gestionar adecuadamente un departamento de mantenimiento sin establecer previamente un sistema que permita atender las necesidades de mantenimiento correctivo (reparación de averías) de forma eficiente. De poco sirven los esfuerzos para tratar de evitar averías si, cuando éstas se producen, dicho departamento no está en capacidad de proporcionar una respuesta adecuada, tomando en cuenta, además, que un alto porcentaje de las horas-hombre dedicadas a estas actividades se emplea en la solución de fallos que no han sido detectados por el personal de mantenimiento, sino sólo comunicados por el personal de producción.

El Instituto Universitario de Tecnología de Cabimas (IUTC), la Universidad Experimental Rafael María Baralt (UNERMB) y la Universidad del Zulia (LUZ), son instituciones dependientes del Ministerio del Poder Popular para la Educación Universitaria, las cuales tienen como función principal contribuir al desarrollo regional e integral mediante la capacitación de recursos humanos en programas de orientación técnica especializada.

Dichas universidades, están autorizadas para otorgar títulos de ingeniería y licenciatura; por ser instituciones educativas públicas, poseen una masa significativa de estudiantes que genera una amplia demanda de instalaciones óptimas para el desarrollo de sus actividades académicasadministrativas.

Estas instituciones públicas están comprometidas a ofrecer dentro de sus instalaciones, no solo conocimientos, sino también garantizar, a su población estudiantil, alimentación, traslado tanto en rutas internas de la ciudad como en rutas externas a ellas, mediante servicios de comedor y transporte así como también servicio de biblioteca para dar acceso a espacios tranquilos donde desarrollar actividades propias del ámbito estudiantil.

En este sentido, para ellas es de vital importancia, entre otras, la gestión del mantenimiento correctivo, como una herramienta de apoyo a la prestación de sus servicios, que permite mejoras en cuanto a eficacia y eficiencia de los procesos en pro de la excelencia operativa al garantizar instalaciones con infraestructura adecuada tanto dentro de las aulas de clases como fuera de ellas, y asegurar que sus actividades 
se lleven a cabo en forma efectiva y que al mismo tiempo aseguren su sostenibilidad.

Entre las diferentes aplicaciones de la gestión de mantenimiento se encuentra la referida a la infraestructura, reconociéndole Corrales (2012), como el andamiaje de un país que permite el desarrollo individual y colectivo de sus habitantes, el cual resulta indispensable para el ejercicio de las actividades económicas además de ser un instrumento insustituible de las políticas dirigidas a disminuir la exclusión social y aumentar el bienestar colectivo. El concepto de infraestructura dentro de la gestión de mantenimiento abarca tres (03) ámbitos como lo son instalaciones (edificaciones y estructuras), carreteras (vialidad), así como también servicios (electricidad, aguas servidas y aguas blancas).

En cuanto al ámbito de instalaciones y servicios, Corrales (2012) afirma que para el año 1980, Venezuela ocupaba un puesto privilegiado a nivel de Latinoamérica en materia de disponibilidad de instalaciones físicas e incluso, no se encontraba muy alejada de la situación de los países del este de Asia. Veinticinco años después, la situación es completamente distinta, pues no se ha dado a las instalaciones de infraestructura la prioridad que amerita, no se ha realizado una gestión de mantenimiento adecuada a las necesidades del país; gracias a esto Venezuela ha perdido sus ventajas competitivas frente a américa latina donde muchos países le alcanzaron y le superan y en estos momentos se sitúa muy por debajo de niveles que ostentan los países asiáticos.

Teniendo en cuenta que, en buena medida, las instituciones de educación universitaria públicas, definen el bienestar colectivo, el grado de cohesión de la sociedad y el nivel de productividad de la economía; esta situación no es diferente para ellas, ya que existen edificios que datan de muchos años, que en otrora conformaron las instalaciones necesarias para la población estudiantil de la época; sin embargo, en la actualidad estas instituciones, en su mayoría, siguen realizando sus actividades académicas y administrativas en las mismas edificaciones construidas desde su fundación, con el agravante de que el presupuesto destinado a las instalaciones se ha reducido lo que genera paralización total de nuevas construcciones y evidentemente, como consecuencia, el deterioro de las edificaciones existentes.

En el caso de las instituciones educativas públicas de la Costa Oriental del Lago, contexto de esta investigación, las cuales conforman la columna vertebral educativa de la región, según entrevistas realizadas al personal, las mismas están obligadas a atender necesidades de estudiantes tanto del sector donde se encuentran ubicadas, como foráneos que buscan obtener una educación de calidad y excelencia.

Sin embargo, hoy día es preocupante la situación en la que se encuentran las instalaciones de estas instituciones ya que hay aulas de clase donde se consigue un alto índice de hongos en techos y paredes debido a la humedad causada por problemas de filtración de aguas blancas y en algunos casos de aguas servidas; existen problemas con las instalaciones eléctricas en todas las instituciones y de manera general en todas las instalaciones, lo cual imposibilita que en varias aulas se puedan desarrollar actividades de forma regular.

La condición antes mencionada trae como consecuencia menos espacio físico para los estudiantes e impide que escuchen clases en las noches debido a falta de iluminación, aun cuando dentro del paquete 
de estudios las instituciones lo ofrecen, lo que al mismo tiempo acarrea sobre población en las aulas de clases durante el día.

También se puede señalar el estado de deterioro de las áreas sanitarias de las instalaciones las que generalmente no tienen servicios de aguas blancas por lo que son poco higiénicas para su uso. Por otra parte las áreas de recreación como los pasillos, caminerías y plazas tienen desniveles que representan un riesgo latente para los transeúntes.

La situación descrita permite afirmar que los espacios mencionados requieren de mantenimiento que preserve sus condiciones y coadyuve a realizar las actividades estudiantiles de forma óptima.

Tomando en cuenta lo anteriormente planteado se formula la siguiente interrogante: ¿Como es la gestión de mantenimiento correctivo en las instalaciones de las universidades públicas de la Costa Oriental del Lago?

\section{MÉTODO}

La presente investigación es de tipo descriptiva, con un diseño no experimental, basado en el diseño de campo, de tipo transeccional. La población estuvo conformada por trabajadores de las distintas casas de estudio, representada por catorce (14) trabajadores de los departamentos de mantenimiento de las universidades públicas de la Costa Oriental del Lago, seis (06) del Instituto Universitario de Tecnología de Cabimas, cuatro (04) de la Universidad Experimental Rafael María Baralt y cuatro (04) de la Universidad del Zulia.

La técnica de recolección de datos utilizada fue la encuesta, y el instrumento utilizado para recabar información fue un cuestionario con escala de medición tipo Likert el cual fue validado mediante el juicio de cinco (05) expertos en metodología y gestión de mantenimiento que dio como resultado una confiabilidad de 0,895 , que lo categorizó como de muy alta confiabilidad.

En cuanto al tratamiento estadístico en esta investigación, fue realizado a través de la estadística descriptiva, mediante las aplicaciones del paquete estadístico SPSS 19, calculando la media aritmética y desviación estándar a los indicadores, así como el porcentaje, frecuencias totales de la variable dimensiones e indicadores en estudio, lo cual permitió deducir información relevante de dichos valores correspondientes, así como tomar decisiones durante la interpretación de los mismos, considerando las escalas y tendencias que muestra el cuadro 1. 
Cuadro 1. Baremo de medición para interpretación de la media

\begin{tabular}{|c|c|c|c|c|}
\hline Valor & Alternativa & Intervalo & Categoría & Descripción \\
\hline 5 & Siempre & $4,21-5,00$ & Muy alto & $\begin{array}{l}\text { Muy alta presencia de desarrollo de la } \\
\text { variable, dimensión o indicador. }\end{array}$ \\
\hline 4 & Casi siempre & $3,41-4,20$ & Alto & $\begin{array}{l}\text { Alta presencia de desarrollo de la } \\
\text { variable, dimensión o indicador, } \\
\text { presentando posibles mejoras. }\end{array}$ \\
\hline 3 & $A$ veces & $2,61-3,40$ & Moderado & $\begin{array}{l}\text { Moderada presencia de la variable, } \\
\text { dimensión o indicador con ciertas } \\
\text { debilidades. }\end{array}$ \\
\hline 2 & Casi nunca & $1,81-2,60$ & Bajo & $\begin{array}{l}\text { Bajo nivel de presencia de la variable, } \\
\text { dimensión o indicador presentando } \\
\text { altas debilidades. }\end{array}$ \\
\hline 1 & Nunca & $1,00-1,80$ & Muy bajo & $\begin{array}{l}\text { Muy bajo nivel de presencia de la } \\
\text { variable, dimensión o indicador podría } \\
\text { decirse que es poco o nada. }\end{array}$ \\
\hline
\end{tabular}

\section{RESULTADOS Y DISCUSIÓN}

A continuación, se muestra la dimensión planificación de mantenimiento, con los siguientes resultados:

Tabla 1. Media de la dimensión planificación de mantenimiento

\begin{tabular}{|c|c|c|}
\hline Indicadores & Media & Categoría \\
\hline Procedimientos & 1,40 & Muy baja presencia \\
\hline Mano de obra & 2,98 & Moderada presencia \\
\hline Materiales, partes y repuestos & 2,52 & Baja presencia \\
\hline Equipos & 2,81 & Moderada presencia \\
\hline $\begin{array}{l}\text { Clasificación del mantenimiento } \\
\text { correctivo }\end{array}$ & 2,14 & Baja presencia \\
\hline Resumen de la dimensión & 2,37 & Baja presencia \\
\hline
\end{tabular}

La tabla 1, muestra los resultados obtenidos para la dimensión planificación de mantenimiento, la cual presenta una media de 2,37 (baja presencia). Se pueden observar oportunidades de mejora en los indicadores procedimientos, mano de obra, equipos y clasificación del mantenimiento correctivo.

Estos resultados difieren con lo expuesto por Perozo (1997), quien afirma que la planificación del mantenimiento consiste en definir en términos cuantitativos y lógicos, todos los elementos de la gestión, su método de aplicación y secuencia de actividades. La planificación de mantenimiento es el proceso de asignación de recursos y personal para los trabajos que tienen que realizarse en determinado momento. El trabajo de mantenimiento para estos equipos se maneja bajo prioridades y es atendido antes de emprender cualquier 
otro trabajo. La ocurrencia de tales trabajos no puede predecirse con certeza, de modo que los programas para el mantenimiento planeado en estos casos deben ser revisados.

En este orden de ideas, se puede afirmar que la planificación del mantenimiento correctivo en las instalaciones de las universidades públicas de la Costa Oriental del Lago opera como el conjunto de actividades que comprende la descripción de las tareas programadas, establecen los procedimientos a realizar, las actividades, los lineamientos y estrategias que conllevan al logro de los objetivos planteados; tomando en cuenta la mano de obra requerida, y los equipos a utilizar, además de la planificación del tipo de mantenimiento correctivo a ejecutar. Se puede evidenciar en esta dimensión oportunidades de mejora en cada uno de los procesos que se generan.

Tabla 2. Media de la dimensión organización de mantenimiento

\begin{tabular}{lcc}
\multicolumn{1}{c}{ Indicadores } & Media & Categoría \\
\hline Mantenimiento correctivo por área & 2,31 & Baja presencia \\
$\begin{array}{l}\text { Mantenimiento centralizado } \\
\text { Mantenimiento correctivo por }\end{array}$ & 2,17 & Baja presencia \\
departamento & 2,21 & Baja presencia \\
\hline \multicolumn{1}{c}{ Resumen de la dimensión } & $\mathbf{2 , 2 3}$ & Baja presencia \\
\hline
\end{tabular}

La tabla 2, muestra los resultados obtenidos para la dimensión organización de mantenimiento, al presentar una media de 2,23 (baja presencia). Se pueden observar oportunidades de mejora en el indicador mantenimiento centralizado.

Estos resultados indican que en las instituciones estudiadas existen grandes fallas en cuanto a la forma como se plantea la organización de los departamentos encargados del mantenimiento. Al respecto, Duffuaa y col. (2010), hacen referencia a que el mantenimiento "se puede organizar por departamentos, por aéreas o en forma centralizada". Cada universidad pública de la Costa Oriental del Lago debería tener un departamento de mantenimiento organizado de acuerdo a su estructura para trabajar de manera centralizada, se observó que en las instituciones tienen talleres centrales para la realización de las reparaciones a fin de dar una rápida respuesta a las necesidades del transporte, sin embargo existen serios problemas en la forma como están estructuradas las áreas para realizar el mantenimiento correctivo, lo cual incide sobre la realización de un trabajo óptimo. 
Tabla 3. Media de la dimensión dirección de mantenimiento

\begin{tabular}{lcc}
\hline \multicolumn{1}{c}{ Indicadores } & Media & Categoría \\
\hline Motivación del recurso humano & 3,31 & Moderada presencia \\
Apoyo logístico & 3,19 & Moderada presencia \\
Apoyo administrativo & 3,14 & Moderada presencia \\
Apoyo gerencial & 2,40 & Baja presencia \\
Manejo de la información & 4,24 & Muy Alta presencia \\
\hline Resumen de la dimensión & $\mathbf{3 , 2 5}$ & Moderada presencia \\
\hline
\end{tabular}

La tabla 3, muestra los resultados obtenidos para la dimensión dirección de mantenimiento, la cual muestra una media de 3,25 (moderada presencia). Se pueden observar que moderadamente se manejan en las instituciones estudiadas los aspectos referidos a motivación del recurso humano, apoyo logístico, apoyo administrativo. Con respecto al apoyo gerencial se observa una baja presencia y una muy alta presencia con relación al manejo de la información.
Estos resultados contradicen lo expuesto por Chiavenato (2004), al exponer que la dirección representa la ejecución de lo que fue planeado y organizado. Es así como la dirección de las universidades públicas de la Costa Oriental del Lago presentan serias debilidades al ejercer la función de dirección $y$ control administrativo que incluye el activar y motivar a las personas para el alcance de los objetivos organizacionales.

Tabla 4. Media de la dimensión control de mantenimiento

\begin{tabular}{lcc}
\hline \multicolumn{1}{c}{ Indicadores } & Media & Categoría \\
\hline Ajuste de la capacidad de mantenimiento & 2,09 & Baja presencia \\
$\begin{array}{l}\text { Ordenes de trabajo } \\
\text { Retroalimentación de la información y }\end{array}$ & 3,44 & Moderada Presencia \\
acción correctiva & 2,28 & Baja presencia \\
Control de inventario & 2,15 & Baja Presencia \\
Indicadores & 2,38 & Baja presencia \\
\hline \multicolumn{1}{c}{ Resumen de la dimensión } & $\mathbf{2 . 4 6}$ & Baja presencia \\
\hline
\end{tabular}

La tabla 5, muestra los resultados obtenidos para la dimensión control de mantenimiento, la que presenta una media de 2,46 (baja presencia). De igual manera se observa baja presencia en los indicadores que conforman la dimensión, lo cual indica que el control del mantenimiento en las instituciones educativas estudiadas presenta grandes debilidades. 
Estos resultados contradicen lo expuesto por Gómez y González (2005), quien indica que las labores propias del mantenimiento correctivo se encuentran apegadas al control, de forma tal que todo es documentado y mantenido para que su eficacia sea continuamente mejorada como lo establece la norma internacional ISO 9000:2000.

Tabla 5. Resultados de la variable gestión de mantenimiento

\begin{tabular}{ccc}
\hline \multicolumn{1}{c}{ Dimensión } & Media & Categoría \\
\hline Planificación de mantenimiento & 2,37 & Baja presencia \\
Organización de mantenimiento & 2,23 & Baja presencia \\
Dirección de mantenimiento & 3,25 & Moderada presencia \\
Control de mantenimiento & 2,46 & Baja presencia \\
\hline Resumen de la variable & $\mathbf{2 , 5 7}$ & Baja presencia \\
\hline
\end{tabular}

La tabla 5, muestra una media de 2,57, lo cual indica una baja presencia de la gestión del proceso de mantenimiento correctivo en las universidades públicas de la Costa Oriental del Lago. Estos resultados contradicen lo expuesto por Pérez (2007), al indicar que las actividades deben medirse con parámetros enfocados en la toma de decisiones para asegurar que vayan en el sentido correcto y permitan evaluar los resultados de la gestión en función de objetivos, metas y responsabilidades.

Finalmente, se puede evidenciar que los resultados de esta investigación coincide con lo expuesto en el planteamiento del problema donde se indica que en la actualidad las instalaciones de las universidades públicas de la Costa Oriental del Lago solo reciben mantenimiento correctivo cuando la falla ocasiona la paralización de sus actividades académicas y/o administrativas, con el agravante de que la reducción del presupuesto destinado a las instalaciones trae como consecuencia no solo la paralización total de nuevas construcciones, sino también el deterioro de las edificaciones existentes por falta de inversión para mantenimiento de las mismas.

\section{DISCUSIÓN}

El nuevo milenio crea un nuevo entorno donde las organizaciones productoras y de servicios se ven presionadas a ser competitivas al ofrecer una entrega oportuna de productos de calidad lo cual compromete a la gerencia y a los ingenieros a optimizar los sistemas que intervienen en sus instituciones; una forma de conseguirlo es mediante el mantenimiento, el cual según Duffuaa y col. (2010) contribuye a reducir los costos, minimizar el tiempo muerto de los equipos, mejorar la calidad e incrementar la productividad; además de contar con un equipo confiable que sea seguro y esté bien configurado para lograr la entrega oportuna de las órdenes de los clientes. La gerencia no solo tiene que minimizar el tiempo muerto sino también controlar de manera eficaz los costos de mantenimiento.

En este sentido Duffuaa y col. (2010), expresan que el mantenimiento correctivo es el conjunto de trabajos encaminados a disminuir la necesidad de mantenimiento, 
basados en el estudio exhaustivo de las averías registradas, condiciones de trabajo de las maquinarias o instalaciones, causas de las averías, duración, costos de reparación y de parada, entre otros. Con todos estos datos se estudian las medidas a tomar para tratar de corregir las fallas, pero esto de una forma integral, es decir, empezando por posibles modificaciones o mejoras en las máquinas, alternativas de proceso e incluso revisión del sistema de mantenimiento existente; se parte de la existencia de un sistema de mantenimiento ya implantado que permite, además, recoger de una forma confiable toda la información citada.

De acuerdo con el autor antes mencionado, el mantenimiento correctivo es aquel que se lleva a cabo después que ocurre una falla y restablece el equipo a un estado en el que puede realizar la función requerida: Se realiza cuando el equipo es incapaz de seguir operando, por lo cual no es un elemento de planificación. Se aplica principalmente en los componentes electrónicos.

De igual manera García (2009), señala que el mantenimiento correctivo es el arreglo de las averías o fallas, cuando estas se presentan. Es la habitual reparación tras una avería que obliga a detener la instalación o máquina afectada por el fallo. El mismo autor sostiene que dicho mantenimiento tiene ventajas porque no genera gastos fijos, desde el punto de vista de quien lo requiere; solo se gasta dinero cuando se está claro que se necesita hacerlo, a corto plazo puede ofrecer un buen resultado económico.

Por su parte, en la norma COVENIN 3049-93 (1993) se estipula el mantenimiento correctivo como aquel que comprende las actividades de todo tipo encaminadas a tratar de eliminar la necesidad de mantenimiento, corrigiendo las fallas de manera integral a mediano plazo. Este tipo de mantenimiento es realizado por el personal de la organización de mantenimiento o por entes foráneos, dependiendo de la magnitud, costos, especializaciones necesarias $u$ otros. Las acciones más comunes que se realizan son modificaciones de elementos de máquinas, modificaciones de alternativas de procesos, cambios de especificaciones, así como la revisión de elementos básicos de mantenimiento y conservación.

De igual manera la norma COVENIN 3049-93 (1993), expresa que la gestión de mantenimiento correctivo es la efectiva y eficiente utilización de los recursos materiales, económicos, humanos y de tiempo para alcanzar los objetivos de mantenimiento. No es posible gestionar adecuadamente un departamento de mantenimiento sin establecer un sistema que permita atender en forma eficiente las necesidades de mantenimiento correctivo (reparación de averías). En este sentido el manual estipula tiempos para la gestión de mantenimiento correctivo que van desde la detección del problema por medio del reconocimiento de los síntomas, causas, solución, medidas preventivas tomadas hasta la redacción del correspondiente informe donde se plasma todo el análisis del caso que pasa a ser un insumo de apoyo en nuevas situaciones al establecer la periodicidad de atención así como dar información sobre materiales y equipos utilizados.

Por su parte Torres (2005), considera que dicha gestión se refiere a actuar sobre todos los aspectos de importancia para el óptimo funcionamiento de una empresa, referidos no únicamente a la reparación de las instalaciones, sistemas o equipos como tal, sino también a los costos que implican 
los recursos humanos y almacenes a fin de desarrollar una óptima gestión.

Según García (2009), gestionar con eficacia el mantenimiento correctivo significa realizar intervenciones con rapidez, que permitan la puesta en marcha del equipo en el menor tiempo posible (tiempo medio de reparación bajo); realizar intervenciones fiables y adoptar medidas para que no se vuelvan a producir estas en un periodo suficientemente largo, (tiempo medio entre fallos grandes) y consumir la menor cantidad de recursos posible (tanto mano de obra como materiales).

Finalmente la gestión de mantenimiento correctivo materializa, en un documento, la forma de operar haciendo tangible la gestión misma; el principal valor para el caso del mantenimiento correctivo es organizarse para evitar que el caos por necesidades que se presentan día a día desenfoquen su gestión. Una vez establecido un sistema de gestión, lo más importante es lograr articularlo, y esta tarea, liderada por el gerente, debe ser acompañada de una adecuada estrategia de comunicación y difusión, así como claras responsabilidades definidas en cada puesto integrante del modelo.

Lo anteriormente planteado permite concluir que, la gestión de mantenimiento correctivo es actuar de manera inmediata sobre todos los aspectos de reparación que ocupen un lugar importante dentro de la organización. Es al mismo tiempo, establecer los tiempos óptimos de espera tomando en cuenta los costos que implican dichas reparaciones con la finalidad de desarrollar una eficiente gestión.

En efecto la gestión de mantenimiento correctivo está referida a todas aquellas acciones orientadas para llevar a cabo el mismo: La planificación, organización, dirección y control de la ejecución de las actividades; las mismas son regidas según una estrategia establecida en términos de medidas administrativas con el propósito de preservar y/o modificar un equipo o instalación para su buen funcionamiento y de esta manera obtener un mayor rendimiento, que resulta en un mayor beneficio económico.

En este orden de ideas la planificación del mantenimiento es definida en términos cuantitativos y lógicos, al expresar el autor que son todos los elementos de la gestión, su método de aplicación y secuencia de actividades (Perozo, 1997). El autor lo concibe, y así lo expresa, como el proceso de asignación de recursos y personal para los trabajos que tienen que realizarse en determinado momento. En la programación de mantenimiento deben tomarse en cuenta los equipos críticos de una planta o proceso productivo, ya que una falla de ellos detendrá el proceso de producción y/o pondrá en riesgo la seguridad de los trabajadores. Este tipo de trabajo de mantenimiento se maneja bajo prioridades y se atiende antes de emprender cualquier otro trabajo.

Según Clemenza (2010), la planificación del mantenimiento es un conjunto de métodos, guías y procedimientos que se requieren para la toma de decisiones. Esto implica que se deben formular una gama de preguntas para lograr que la planificación sea exitosa.

En este sentido Duffuaa y col. (2010), señalan que la planificación en el contexto de mantenimiento se refiere al proceso mediante el cual se determinan y preparan todos los elementos requeridos para efectuar una tarea antes de iniciar el trabajo. El proceso de planificación comprende todas las funciones relacionadas con la preparación 
de la orden de trabajo, la lista de materiales, la requisición de compras, los planos y los dibujos necesarios, la hoja de planeación de la mano de obra, los estándares de tiempo y todos los datos necesarios antes de programar y liberar la orden de trabajo.

Es así como se puede definir la planificación del mantenimiento como el conjunto de actividades que comprende la descripción de los objetivos de una organización, establecer los procedimientos a realizar, las actividades, lineamientos y estrategias que conllevan al logro de los objetivos planteados; sin olvidar tomar en cuenta la mano de obra requerida, los materiales, partes y repuestos necesarios además de los equipos a utilizar.

La planificación del mantenimiento constituye el punto de partida de la gestión ya que involucra la necesidad de visualizar y relacionar las actividades que habrán de cumplirse para obtener como resultado los objetivos planteados, sin pasar por alto los recursos necesarios para alcanzarlos. El estudio de esta dimensión del mantenimiento correctivo involucra conocer los elementos que la componen como son: Procedimientos, mano de obra, materiales, partes y repuestos, equipos y clasificación del mantenimiento correctivo.

Para Clemenza (2010), los procedimientos consisten en saber qué y cómo se va a hacer, cual es el sistema, aparato o equipo que se intervendrá, con qué seguridad se hará, cuál será el procedimiento técnico, con cual manual. De igual manera Villarroel (2005) explica que, los procedimientos son la aplicación secuenciada de las tareas con sus respectivas relaciones que especifican su ejecución; deben contar con un nivel de detalle que permita analizar sus posibles mejoras además la secuencia de las tareas están normadas y alineadas con la estructura de la organización. Su característica principal es el nivel de detalle que entrega de las acciones que se lleva a cabo.

En cuanto a la mano de obra Clemenza (2010) expresa que ésta se define al conocer su tipo y cantidad, cuánto tiempo por tipo de mano de obra, cantidad total de tiempo y que secuencia se utilizará. Esta misma autora expresa que con relación a materiales, partes y repuestos se debe especificar el tipo de insumos, la cantidad de los mismos, además de indicar cual tipo de materiales, partes y repuestos se requieren.

Otro elemento a considerar dentro de la planificación del mantenimiento correctivo es el equipo; en este sentido, Clemenza (2010) expresa que lo más importante consiste en especificar cuáles herramientas y equipos utilizar, del equipo soporte, así como el tiempo de uso de los mismos.

Finalmente también forman parte de la planificación, los tipos de mantenimiento correctivo. Para García (2009), existen dos (02) formas diferenciadas: El programado y el no programado. La diferencia entre ambos radica en que mientras el no programado supone la reparación de la falla inmediatamente después de presentarse, el mantenimiento correctivo programado o planificado supone la corrección de la falla cuando se cuenta con el personal, las herramientas, la información y los materiales necesarios. La decisión entre corregir un fallo de forma programada o de forma inmediata, la define la importancia del equipo en el sistema productivo.

Por su parte Duffuaa y col. (2010) expresan que el mantenimiento correctivo puede dividirse en ambas categorías: mantenimiento planeado y mantenimiento no planeado. El mantenimiento correctivo implica, en primer lugar, que todos los 
recursos necesarios para realizar las tareas han sido planeados previamente incluyendo su disponibilidad y en segundo lugar, que el trabajo se llevara a cabo de acuerdo con un programa establecido.

Otro componente de la gestión del mantenimiento correctivo es la organización, sobre este aspecto Duffuaa y col. (2010), hacen referencia a la posibilidad de organizar el mantenimiento por departamentos, por áreas o en forma centralizada. Enfatiza que cada tipo de organización tiene sus pros y sus contras. En organizaciones grandes descentralizar la función de mantenimiento puede producir un tiempo de respuesta más rápido y lograr que los trabajadores se familiaricen más con los problemas de una sección particular de la planta. Sin embargo crear un número de pequeñas unidades tiende a reducir la flexibilidad del sistema de mantenimiento. La gama de habilidades disponibles se reduce y la utilización de la mano de obra es menor que en una unidad de mantenimiento centralizada.

En conclusión, organizar el mantenimiento correctivo es una realidad compleja y única para cada tipo de organización así como para cada tipo de trabajo, abarca desde la problemática estrictamente técnica hasta diversos tipos de efectos humanos, también sociales; lo cual implica una organización definida para poder cumplir a cabalidad sus funciones sin inconvenientes.

A tal efecto, la norma COVENIN 3049-93 (1993), establece que el mantenimiento correctivo por área subdivide a la organización en varias partes geográficas y a cada una de ellas se asignan cuadrillas de personal para ejecutar acciones de mantenimiento. Su objetivo es aumentar la eficiencia operativa, al situar pequeñas organizaciones próximos a los sistemas a los cuales sirven. Se caracteriza por mejor control de personal por área, personal especializado en el área de trabajo, aumento de costos por especialización funcional, mayor fuerza laboral, programación, además de prevenciones más ajustadas a la realidad y sistemas de información más complejos.

En este mismo orden de ideas la norma COVENIN 3049-93 (1993) establece que el mantenimiento centralizado, es la concentración de los recursos de mantenimiento en una localización central. Se caracteriza por la movilización del personal que tiene el conocimiento del sistema de producción a mantener, el bajo nivel de especialización en general comparado con el de área, la reducción de costos por la poca especialización funcional; lo que permite que en emergencias se puede contar con todo el personal; se recomienda para sistemas de producción medianos a pequeños con poca diversidad de procesos.

Según García (2009), el mantenimiento centralizado, es la estructura organizacional en la que los administradores mantienen un elevado grado de autoridad al más alto nivel o cerca del mismo; sus ventajas son varias: las decisiones son tomadas por administradores que poseen una visión global de la empresa, quienes deciden suelen estar en posiciones jerárquicas más elevadas, por lo que suele ser personal mejor entrenado en comparación con quienes ocupan lugares más bajos.

Para Duffua y col. (2010), las organizaciones de mantenimiento centralizadas, se caracterizan por tener un personal transferible de un área a otra, controlado desde una localización central, los talleres son centralizados, tienen menores problemas de transporte, prioridades, compras, entre otras y requieren de un estricto control de costos. 
En efecto, para los autores el mantenimiento centralizado es concentrar todos los recursos destinados al mantenimiento en un departamento o cede central, de forma tal que el personal se pueda movilizar en base a su nivel de especialización, con la finalidad de poder mantener el sistema de producción operativo.

Asimismo, la norma COVENIN 3049-93 (1993) establece que el mantenimiento correctivo por departamentos, solo se aplica en macro sistemas de producción, los cuales tienen sede en situaciones geográficas alejadas, cantidades elevadas de personal y diversidad de procesos. En este tipo de entes organizacionales cada área tiene su organización de mantenimiento, pero todas manejadas bajo una administración central.

La dirección de mantenimiento es otro elemento de la gestión de mantenimiento correctivo que según expresa Chiavenato (2004), representa la puesta en marcha de lo que fue planeado y organizado. En consecuencia, la dirección es la función administrativa que incluye el influir sobre el personal para activarlos y motivarlos a alcanzar los objetivos organizacionales. Se encarga de comunicar las tareas esenciales a ser ejecutadas. La dirección es el proceso de influir y orientar como un todo las actividades relacionadas con las tareas de los diversos miembros del equipo o de la organización.

En efecto, la norma COVENIN 2500-93 (1993), expresa que existen mecanismos de incentivos y motivación del recurso humano para mantener el interés y elevar el nivel de responsabilidad del personal en el desarrollo de sus funciones. Es importante resaltar que, el proceso de dirección del mantenimiento debe contemplar el recurso humano como el factor más importante para el desarrollo de las funciones de planeación, organización y control, dentro del mantenimiento, por lo cual es necesario mantener un personal altamente motivado; la empresa debe ejecutar programas de capacitación que permitan la mejora continua del personal, otorgar incentivos o estímulos basados en la puntualidad, asistencia al trabajo, calidad de trabajo, iniciativa, sugerencias para mejorar el desarrollo de la actividad de mantenimiento correctivo y propiciar un ambiente de armonía donde la comunicación eficaz sea el punto de partida para las relaciones que se dan en el departamento.

Se considera pues, importante poseer un sistema de información con el que la comunicación entre los involucrados en la gestión de mantenimiento correctivo y el resto de la empresa fluya conforme a las necesidades.

Asimismo, en cuanto al apoyo logístico, la norma COVENIN 2500-93, expresa que la organización de mantenimiento correctivo debe poseer un sistema de evaluación periódico del trabajador, para poder asignarle merecido incentivo de acuerdo a la apreciación de sus capacidades y resultado de sus labores.

De igual manera, así como la organización de mantenimiento correctivo debe tener un sistema de evaluación periódica del trabajador para fines de ascensos o aumentos salariales, también debe contar con el apoyo administrativo de la organización en cuanto a recursos humanos, financieros y materiales, los cuales deben ser suficientes para cumplir los objetivos trazados por la organización. Según Hitt y col. (2006), toda organización dentro de su administración debe contar con un apoyo que le permita estructurar y utilizar un conjunto de recursos orientados al logro de las metas, para de esta forma llevar a cabo 
las tareas en un entorno organizacional estable.

En este mismo orden de ideas, la Norma COVENIN 2500-93 (1993), expone que la gerencia debe estar informada sobre la situación y el desarrollo de los planes de mantenimiento correctivo formulados por el ente de mantenimiento, permitiendo así asesorar la misma, en cualquier situación que atañe a sus operaciones. La gerencia le da al mantenimiento la misma importancia de las unidades principales en el organigrama funcional de la empresa.

Es así como a criterio de Davis y col. (1994) los sistemas de apoyo gerencial son una colección de sistemas de información que interactúan entre sí y que proporcionan información tanto para las necesidades de las operaciones como de la administración.

En cuanto al manejo de la información la norma COVENIN 2500-93 establece como principio básico que la empresa debe contar con una estructura técnica administrativa para la recolección, depuración, almacenamiento, procesamiento $\mathrm{y}$ distribución de la información que el sistema productivo requiera, así como con mecanismos para evitar introducir información errada o incompleta en el sistema de información; debe poseer un archivo ordenado, así como jerarquizado técnicamente.

Del mismo modo, precisa procedimientos normalizados (formatos) para llevar y comunicar la información entre las diferentes secciones o unidades, así como almacenamiento (archivo) para su cabal recuperación. Finalmente, la información debe procesarse en base a los resultados que se deseen obtener, al igual que contar con mecanismos que hagan llegar la información recopilada y procesada a las personas que deban manejarla.
Por su parte, Duffuaa y col. (2010) resaltan el énfasis de la acción de la dirección sobre la organización, en todos los aspectos que la conforman: Personal, mano de obra, motivación, administración, instalaciones, control de almacenes materiales y herramientas y en los sistemas de información; por el impacto significativo que tienen en el sistema de mantenimiento correctivo.

De la misma manera, Gómez y González (2005), plantean que la esencia del control del mantenimiento correctivo radica en asociar las actividades y labores propias del mantenimiento correctivo a los estándares fijados para tal fin, como es el caso de la norma internacional ISO 9000:2000, la cual exige a las organizaciones que quieran implementarla, la identificación de los procesos necesarios para el sistema de gestión de mantenimiento correctivo y su aplicación a través de la organización, el determinar criterios y métodos necesarios para asegurar que tanto la operación como el control de los procesos sean eficaces e igualmente deben asegurar la disponibilidad de recursos e información necesarios para apoyar la operación y el seguimiento de los procesos.

Difieren de esto Duffuaa y col. (2010), quienes opinan que el control, debe evaluarse como el porcentaje de trabajos de mantenimiento aceptados de acuerdo a la norma adoptada por la organización. Se ejerce midiendo los atributos del producto o servicio y comparando estos con sus respectivas especificaciones. Mencionan las órdenes de trabajo, definidas como una forma donde se detallan las instrucciones escritas para el trabajo que se va a realizar, como la fuente principal de información para el control del mantenimiento. Según Duffuaa y col. (2010), la función de coordinación de 
las órdenes de trabajo es planear y coordinar los diferentes tipos de solicitudes de mantenimiento con base en las restricciones de operación, la disponibilidad de recursos y la prioridad. Debido a la extensa cantidad de órdenes de mantenimiento que se necesitan tanto en el trabajo de mantenimiento no planificado como en el planificado, a menudo resulta imposible obtener una distribución equilibrada de la carga de todos los puestos de trabajo de mantenimiento disponibles en el momento de la creación de la orden, por lo que se hace necesario que el planificador de mantenimiento realice periódicamente un ajuste de la capacidad de mantenimiento.

En efecto, las ordenes de trabajo Según Dufuaa y col. (2010) son básicamente una herramienta documentada para la organización que permite realizar determinada actividad, ellas especifican que se va a realizar y como se realizará; es el primer contacto para obtener un bien o servicio de quien lo presta.

Por su parte González (2005), indica que la optimización del mantenimiento, en especial el mantenimiento preventivo rutinario y el correctivo, se fundamenta en sistemas de planificación y control de los trabajos que deben ser efectivos, que permitan llevar el control de las órdenes de trabajo emitidas así como el conocer el avance de las que están en proceso.

La retroalimentación de la información y acciones correctivas, es otro aspecto que según Duffuaa y col. (2010), se trata esencialmente de la recopilación de información así como la toma de decisiones para alcanzar las metas y objetivos establecidos, al indicar el curso de acción apropiado, encaminado a mejorar el control del trabajo, de inventarios, de los costos y de la calidad.
Dentro del marco del mantenimiento, el control de inventario de partes y repuestos es importante tanto para la planificación de las actividades de revisión y reparación de los equipos, como para la gestión de los costos. Tal como lo señala Duffuaa y col. (2010), la planeación de los requerimientos de materiales y el control de inventarios son decisivos para la función global del sistema de mantenimiento, mediante el uso de sistemas de información que provean al personal responsable del mantenimiento, detalles como la disponibilidad en cantidad y ubicación de las piezas a sustituir, el progreso de las ordenes de trabajo y la posibilidad de realizar la clasificación de los repuestos de acuerdo a criterios de frecuencia de uso y costos.

De igual manera los indicadores de gestión son de gran importancia en la gestión de mantenimiento correctivo. Según Serna (2003) se entiende por indicador al conjunto de variables cuantitativas o cualitativas que se van a monitorear y medir, Las diferentes aéreas funcionales de las organizaciones deben contar con indicadores que formen parte del sistema de control, con los que proporcionan información oportuna sobre los aspectos importantes de un proceso critico o del conjunto de actividades que desarrolla la organización, al correlacionar los datos, permitiendo la toma de decisiones.

Entre los indicadores utilizados para medir los resultados de gestión están los indicadores de eficacia, de cumplimiento, de confiabilidad, de efectividad, de satisfacción al cliente y de disponibilidad. Los indicadores de gestión tienen el objetivo de con base en su cumplimiento en forma organizada, valorar los resultados alcanzados en función de los objetivos planteados en la organización, Fleitman (2007). 


\section{CONCLUSIONES}

Los resultados llevan a concluir que en las instituciones educativas objeto de estudio de esta investigación la planificación del mantenimiento correctivo no opera en forma eficiente, observándose oportunidades de mejora en cada uno de los procesos que la componen.

En cuanto a la dimensión organización de mantenimiento se concluye que existen grandes fallas en la forma como se plantea la organización de los departamentos encargados del mantenimiento en cada universidad pública de la Costa Oriental del Lago, se considera conveniente la restructuración de sus talleres, para el mantenimiento correctivo, en función de las áreas de servicio, lo cual incidiría sobre la realización de un trabajo óptimo y la disminución de reclamos.

Igualmente, con respecto a la dimensión dirección de mantenimiento, se apreció en las instituciones en estudio un moderado manejo de la motivación del recurso humano, apoyo logístico, apoyo administrativo, con oportunidad de mejora en cuanto al apoyo gerencial lo cual contrasta con el manejo de la información donde se observó una muy alta presencia

Es así como se concluye que la dirección de las universidades públicas de la Costa Oriental del Lago presenta serias debilidades en el ejercicio de la función directiva.

En relación a la dimensión control de mantenimiento, los resultados evidenciaron que en las instituciones educativas estudiadas este aspecto presenta grandes debilidades lo que valida lo expresado por autores como Pérez (2007) cuando indica que las actividades deben medirse, controlarse, con parámetros enfocados en la toma de decisiones para asegurar que vayan en el sentido correcto y permitan evaluar los resultados de la gestión en función de objetivos, metas y responsabilidades.

Finalmente, se puede evidenciar que los resultados de esta investigación coinciden con lo expuesto en el planteamiento del problema donde se indica que en la actualidad las instalaciones de las universidades públicas de la Costa Oriental del Lago solo reciben mantenimiento correctivo cuando la falla ocasiona la paralización de sus actividades académicas y/o administrativas, con el agravante de que la reducción del presupuesto destinado a las instalaciones trae como consecuencia, no solo la paralización total de nuevas construcciones, sino también el deterioro de las edificaciones existentes por falta de inversión para mantenimiento de las mismas, por lo cual se puede concluir una baja presencia de la gestión del proceso de mantenimiento correctivo, variable en estudio, en las universidades públicas de la Costa Oriental del Lago.

\section{REFERENCIAS}

Chiavenato, I. (2004). Administración: teoría, proceso y práctica.: McGraw-Hill Interamericana. Bogotá, Colombia

Clemenza, B (2010). Como desarrollar e implantar un sistema de mantenimiento. Editorial Astro Data S.A. 1era edición.

Corrales, M. (2012). Infraestructura pública y servicios asociados. Universidad Católica Andrés Bello (UCAB). Caracas

Davis, Gordon M y Olson, Margrethe (1994). Sistemas de Información Gerencial. Editorial McGraw-Hill $2^{\circ}$ Edición

Duffua y Col (2010). Sistemas de mantenimiento planificación y control. Editorial Limusa. México

Fleitman, J. (2007). Evaluación integral para evaluar modelos de calidad. México. Editorial México

García, S. (2009). Mantenimiento correctivo, Organización y gestión de la reparación 
de averías. Volumen 4. Editorial Renovetec. Caracas. Venezuela

Gómez y González. (2005). Tecnología del mantenimiento industrial. España: Universidad de Murcia

González, J. (2005). Teoría y práctica del mantenimiento industrial. Madrid: fundación Confemetal

Hitt M., Black S. y Porter L. (2006). Administración. 1era Edición. Editorial Pearson.

NORMA VENEZOLANA COVENIN 3049-93. Mantenimiento

NORMA VENEZOLANA COVENIN 2500-93 (1993)

Normas COVENIN-ISO 9000. Sistemas de gestión de la calidad. Fundamentos y vocabulario
Perozo, A. (1997). Mantenimiento preventivo, predictivo y correctivo, guía práctica para estudiantes. Maracaibo. Estado Zulia

Serna, H. (2003). Gerencia estratégica. Bogotá. Ediciones Global

Torres, A. (2005). Mantenimiento industrial. 3era edición

Villarroel, C (2005) Gerencia, Planificación y Evaluación Universitarias. Artículo publicado en la revista Educere, octubre-diciembre, año 9/Vol 9, número 031. Universidad de los Andes. Mérida Venezuela 